\title{
CRANIAL ULTRASOUND AND MRI IN PRETERM INFANTS
}

Cranial ultrasound (cUS) and conventional MRI, performed on the same day at term age, were compared in a population-based cohort of 72 extremely low gestational age (ELGA) infants (below 27 weeks) in a study at the Karolinska Institute, Stockholm, Sweden; and Sophia Children's Hospital, Rotterdam, The Netherlands. MRI findings: MRI was normal or showed only mild abnormalities in $83 \%$ of infants, and $17 \%$ had moderate or severe white matter (WM) abnormalities. No WM abnormalities were detected on MRI in 31/72 (43\%) infants; abnormalities were mild in 29 (40\%), moderate in $9(13 \%)$, and severe in $3(4 \%)$. Grey matter abnormalities were found in $8(11 \%)$ infants, 4 having a small cerebellar hemorrhage. Ultrasound findings: US was normal in $28(39 \%)$, abnormalities were mild to moderate in $41(57 \%)$, and severe in $3(4 \%)$ infants. MRI and US compared: All 3 infants with severe abnormalities were scored the same on MRI and US. Of 28 infants with normal US, 18 (64\%) had completely normal MRI, and $10(36 \%)$ had only mild WM abnormalities on MRI. Normal MRIs in 10 infants were scored as mild to moderate abnormality in cUS. Cerebellar hemorrhages in 4 infants diagnosed by MRI were not detected on cUS. (Horsch S, Skiold B, Hallberg B, et al. Cranial ultrasound and MRI at term age in extremely preterm infants. Arch Dis Child Fetal Neonatal Ed Sept 2010;95:F310-F314). (Respond: Dr Sandra Horsch, Dr Molenwaterplein 60, Rotterdam 3015GJ, The Netherlands. E-mail: s.horsch@ gmx.de).

COMMENT. US and MRI performed on the same day at term age in ELGA infants were equally effective in detecting moderate or severe white matter abnormalities. MRI was modestly superior to US in diagnosis of mild abnormalities. Ultrasound screening test will identify infants with significant severe cranial abnormalities, and conventional MRI may provide additional relevant information regarding mild abnormalities, not always apparent on cUS. MRI spectroscopy and functional MRI are expected to improve the information obtained by conventional MRI.

Previous studies find conventional MRI to be superior to cUS in predicting adverse neurodevelopmental outcome at 2 years. Some authorities advocate the introduction of MRI as a screening test in all ELGA infants. The present authors emphasize the utility of the cUS as a screening test and the importance of comparing the diagnostic sensitivity of US and MRI on the same day at term age.

\section{VASCULAR DISORDERS}

\section{STROKE AFTER VARICELLA-ZOSTER INFECTION}

Researchers at University Hospitals of Ferrara and Padova, Italy, report a case of cerebral infarction and stroke occurring 3 months after varicella-zoster virus (VZV) infection in a 5-year-old immunocompetent child, and they provide a literature review of 70 similar cases. The child was admitted with a left hemiparesis of 10 days duration and gradual onset without fever or impaired consciousness. She had a past history of chickenpox at age 1 year and reactivation of VZV infection affecting the trunk 3 months before admission. Laboratory tests for other causes of stroke were negative. MR angiography showed narrowing of the right middle cerebral artery, compatible with 
infarction and vasculitis. CSF showed a protein of $98 \mathrm{mg} / \mathrm{dL}$, and PCR evidence of VZVDNA. Heparin and acyclovir were started and 10 days later, aspirin $(50 \mathrm{mg} / \mathrm{day})$ to prevent recurrence of infarction. The paresis improved and at 1-year follow-up, she had minimal reduction in motility of the left arm and leg during sports. MRI showed punctate gliosis and malacia in right semiovale.

A total of 70 published cases of childhood stroke syndrome associated with VZV are reviewed, median age 5 years (range 6 months- 11 years), 43 boys and 28 girls; 10 patients $(14 \%)$ had underlying diseases or prothrombotic conditions (diabetes, AIDS, patent foramen ovale, iron deficiency anemia, protein C deficiency) and $61(86 \%)$ were previously healthy and without risk factors for stroke. Stroke was preccded by chickenpox in $90 \%$ cases and by herpes zoster in 10\%, mainly ophthalmic HZ. In 2 cases, stroke followed vaccination with VZV, and in one at birth secondary to maternal $\mathrm{HZ}$ in the $3^{\text {rd }}$ trimester of pregnancy. Median interval between chickenpox or $\mathrm{HZ}$ and stroke was 18 weeks (range, $0-4$ years). Stroke preceded chickenpox exanthema by 24 hours in 1 case and 2 weeks after viral exposure. Role of treatment is unclear (no treatment in $17 \%$, aspirin in $52 \%$, acyclovir $44 \%$, corticosteroids $40 \%$, heparin $15 \%$ ) and outcome is usually good, with complete recovery in 50\%, including those untreated. (Ciccone S, Faggioli R, Calzolari F, Sartori S, Calderone M, Borgna-Pignatti C. Stroke after varicella-zoster infection. Report of a case and review of the literature. Pediatr Infect Dis J Sept 2010;29(9):864-867). (Respond: Dr Sara Ciccone, Clinica Pediatrica, Universita di Ferrara, 44100 Ferrara, Italy. E-mail: $\underline{\text { saraciccone.unife@ gmail.com). }}$

COMMENT. History of recent chickenpox or $\mathrm{HZ}$ should be considered as a possible cause in a child presenting with ischemic stroke. This report adds support for universal immunization against VZV.

Dr Charles Grose, University of Iowa, who had reviewed all the literature on the complications of varicella infection in children in 1994, reports that stroke was rarely mentioned (Pediatr Infect Dis J 2010;29:868-869). MRI is the reason for the emergence of more case reports of stroke after varicella in the past 2 decades. Stroke after zoster infection is more likely if the location is the eye (zoster ophthalmicus). VZV infects the trigeminal ganglion and other cerebral ganglia, and later reactivates, spreading along sensory nerve fibers to an eye and a cerebral artery, where viral replication causes inflammation and thrombosis. Treatment of zoster ophthalmicus with acyclovir is recommended. Neurological complications of VZV have been underestimated.

\section{CEREBRAL COMPLICATIONS OF HYPERTENSION}

Acute and chronic neurological complications of hypertension in patients younger than 21 years were reviewed in the literature, 1980-2010, by researchers at Maimonides Childrens' Hospital, State University and Mount Sinai School of Medicine, New York; and UCL Institute of Child Health, London, UK. Childhood hypertension is underdiagnosed and is defined as an average pressure above the $95^{\text {th }}$ percentile for sex, age, and height on 3 occasions. In a series of 212 pediatric patients with arterial ischemic stroke, systolic BP at immediate follow-up was $>90^{\text {th }}$ percentile in $54 \%$ with symptomatic stroke and in $46 \%$ with cryptogenic stroke. Children with moya-moya disease and stroke were 3-4 times more likely to have hypertension than those with normal vessels. Masked 